\title{
Comparison of protons, carbon and fullerene impacts on a carbon cylinder
}

\author{
R.P. Webb *, I.H. Wilson \\ Department of Electronic Engineering, Chinese University of Hong Kong, Hong Kong
}

\begin{abstract}
The use of protons in radiation treatment has been much studied and is widely used in the treatment of cancer cells. The proton causes damage to the strands of DNA in the cells largely by interaction with the electronic system. The proton interactions largely result in bond breakage in the DNA molecules. Sometimes the resulting damage to the DNA molecule is insufficient to cause both strands of the molecule to be broken and consequently the cell can reproduce despite being irradiated. Heavy ion therapy using carbon ions has been used to try and introduce more damage into the molecule structure, ensuring that both strands of the DNA are broken and hence preventing the cell from replicating. However, it seems that even the use of ions as heavy as carbon still result in DNA that is not fully damaged.

The simulations reported here form a preliminary investigation on the possible use of a molecular species to deliver multiple impacting particles to the molecular structure thereby increasing the energy density of the impacts and thereby concentrating the damaging process to ensure the DNA molecule will be completely broken.

The DNA molecule itself is a very difficult molecule to simulate in a full many body molecular dynamics simulation environment and in this conceptual study we approximate the DNA molecule by a set of carbon discs and investigate the difference between the impacts of protons, carbon atoms and fullerene molecules on an elongated strong of carbon discs. The cylinder used has a similar atom density and radius to DNA and the equilibrium disc separation is very similar to the separation distance of the individual nucleotides found in DNA, consequently this much simpler system might be considered to be a reasonable starting point for a thought experiment to see if there are any potential advantages in using molecular or cluster species in this form of treatment.
\end{abstract}

(c) 2002 Elsevier Science B.V. All rights reserved.

\section{Introduction}

Radiation therapy in the treatment of tumours is well used around the world and there are many

\footnotetext{
${ }^{*}$ Corresponding author. Present address: School of Electronic Engineering, Computing and Mathematics, University of Surrey, Guildford, Surrey GU2 7XH, UK.

E-mail address: r.webb@eim.surrey.ac.uk (R.P. Webb).
}

articles and web pages devoted to this topic which can give the reader a lot more information [1-4].

Conventionally X-rays are used as the doses and energies required for treatment are relatively simple to produce. However in the treatment of tumours in sensitive parts of the body the damage process of an X-ray is such that it is difficult to irradiate a buried tumour without severely effecting neighbouring tissue and complex tomographic 
systems need to be used. For a number of years proton therapy has been employed in many of these more difficult cases because the damage profile - dominated by the well known Bragg peak - induced by a high energy proton beam is much better suited to treat these type of tumour - it is a factor 5 lower in the surface compared to its deep peak value.

The investigation reported here was "inspired" by a workshop session on "the Biological Applications of Ion Beams" held as part of the ECAART 7 conference [5] held at the University of Surrey in August 2001. As part of a general discussion [6] on the use of ion beams in the treatment of tumours it was noted that fluences for radiation treatment of tumours is higher than would be expected based purely on the fluence needed to damage every cell in the tumour. This is thought to be because to prevent a cell from replicating itself, the DNA in the cell's nucleus must be destroyed. However, when the DNA is struck by an energetic particle sometimes it does not damage the DNA sufficiently to prevent either repair or splitting and hence reproduction. For example, if a DNA molecule is struck by an energetic proton it might only suffer damage to one half of the double helical structure of the DNA, still allowing the molecule to be replicated from the still intact half. The major damaging mechanism in radiation therapy is via a bond breaking mechanism generated by the electronic slowing down of the particle, rather than from nuclear displacement due to direct nuclear displacement. The light ion irradiation causes a cascade of electrons during the slowing down process and it is this which is thought to be primarily responsible for the damage caused to the DNA [7]. The cascading electrons break bonds in the DNA, if the intensity of the cascade is high enough it will cause multiple breaks in the DNA molecule. However, the damage so created could be relatively simple to repair if the number of bonds broken in the same area is not high. Hence even though a DNA molecule may undergo typically 50-100 "breakages" during the passage of a single light ion [8] it can still survive the ordeal intact enough to reproduce itself.

The purpose of the rather simplistic work presented here is to look at the feasibility of em- ploying a molecular species to severely damage the DNA molecule in collision. In the case of heavier ion irradiation, such as carbon ions, the creation of cascading electrons can be more efficient [9] and the ion nucleus itself can cause substantial damage to the DNA molecule itself through direct collision with the constituent atoms. Here we explore the possibility of employing molecular ion species to further increase the energy density available to direct collision between the atoms and nuclei of the energetic particles. The damage created by such interactions will be far more substantial than the simple bond breaking caused by the interaction of energetic protons. We use a simple model to conduct a "thought experiment" to see if this might give an improved scheme.

\section{The model}

The model used here is quite simplistic but incorporates the essence of the problem to explore if there might be any benefit in using molecular species in radiation treatment. The model employs a full molecular dynamics programme, which has been described in detail in the past [10], employing realistic many body potentials. These are readily available for the $\mathrm{H}-\mathrm{C}$ system [11] and include the long distance interlayer binding in graphite [12], but are less available for particularly $\mathrm{O}$ and $\mathrm{N}$. As the object here was to investigate the relative behaviour of $\mathrm{H}, \mathrm{C}$ and molecular $\mathrm{C}$ with a structure at least similar to that of DNA we construct a carbon disc structure - essentially a graphite lattice cut into discs each containing 42 atoms with a spacing of 3.5 Angstroms. For what appears to be such a poor model of DNA at first sight does actually have many similar properties to the real thing! Surprisingly the atom density turns out to be very much the same as DNA, the width of the strand is also about the same size and DNA is often modelled as a disc structure (each disc being a nucleotide) with a separation of about 3.5 Angstroms [13]. The familiar helical picture of DNA is not represented here, the backbone of which consists of a phosphate chain, however for the purposes of this investigation the density of particles is more important than the precise locations of the 
atoms themselves. Also DNA is wrapped up in the cell in a "spaghetti like" ball, but straight segments of the DNA are quite long (of the order a few hundred Angstroms [13]) and the bends are such that the next DNA strand is a substantial distance away from any other, so modelling a single strand segment should also be a reasonably good approximation. So even though all the right atoms are not in all the right places we feel the model might suffice for this initial investigation.

\section{Results}

The following demonstrates what happens when various ion species interact with this carbon cylinder and the possible benefits of using a molecular impacting species is explored. The pictures shown are typical of a small - 35 - sample of individual impacts on the carbon cylinders.

The pictures in Fig. 1 show a time sequence of the interaction of a $200 \mathrm{eV}$ proton with the carbon cylinder. From just the dynamics of the nuclear motion of the interacting atoms very little substantial happens other than that one of the graphite disks "bends". Even at $2 \mathrm{keV}$ the proton

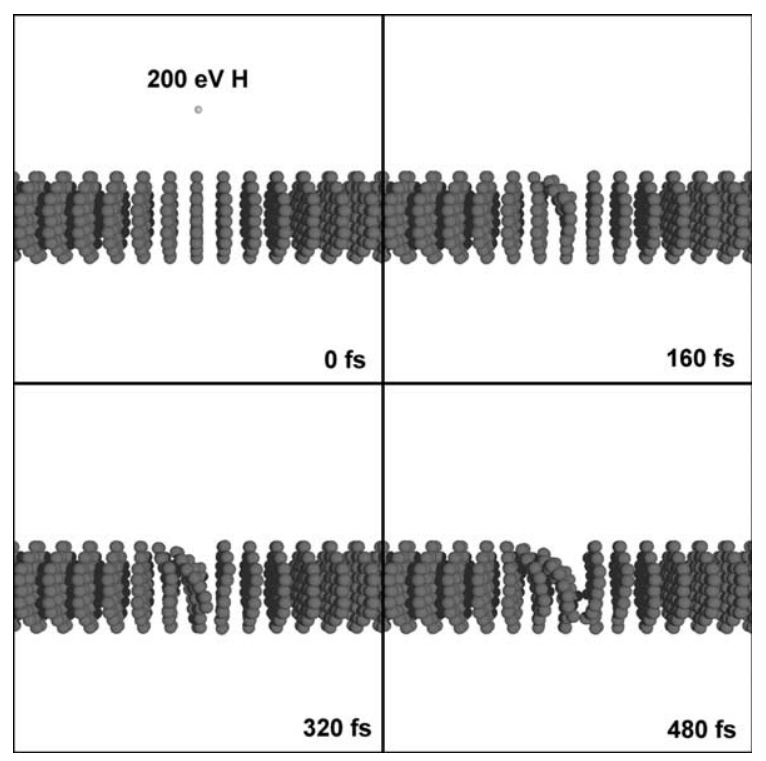

Fig. 1. Time sequence of the interaction of a $200 \mathrm{eV}$ proton with a graphite cylinder. tends to just bounce from the structure, causing even less movement in the carbon cylinder itself. It is quite difficult for the proton to cause substantial damage to the structure by direct nuclear displacement. As mentioned earlier the principal damaging mechanism from proton irradiation is through the electronic energy loss process, causing bond breaking. In this situation the internal stresses of the molecule are critical in determining the stability and consequences of this process. In the case of proton irradiation it is quite clear that the induced molecular damage is very heavily dominated by this process and that direct nuclear displacement is almost inconsequential. As mentioned previously the potential disadvantage of this is that bond breaking can be more easily repaired and can lead to only single strand breaks and therefore leave the cell still viable. The consequence of this is that the treatment dose needs to be higher than might initially be expected.

Fig. 2 shows the effect of a $2 \mathrm{keV} \mathrm{C}$ atom hitting the structure. A $2 \mathrm{keV} \mathrm{C}$ has a similar velocity to a $200 \mathrm{eV}$ proton. The damage is certainly more substantial and 3 atoms are physically ejected from the system. The remaining discs of the system, although severely agitated, will probably recover

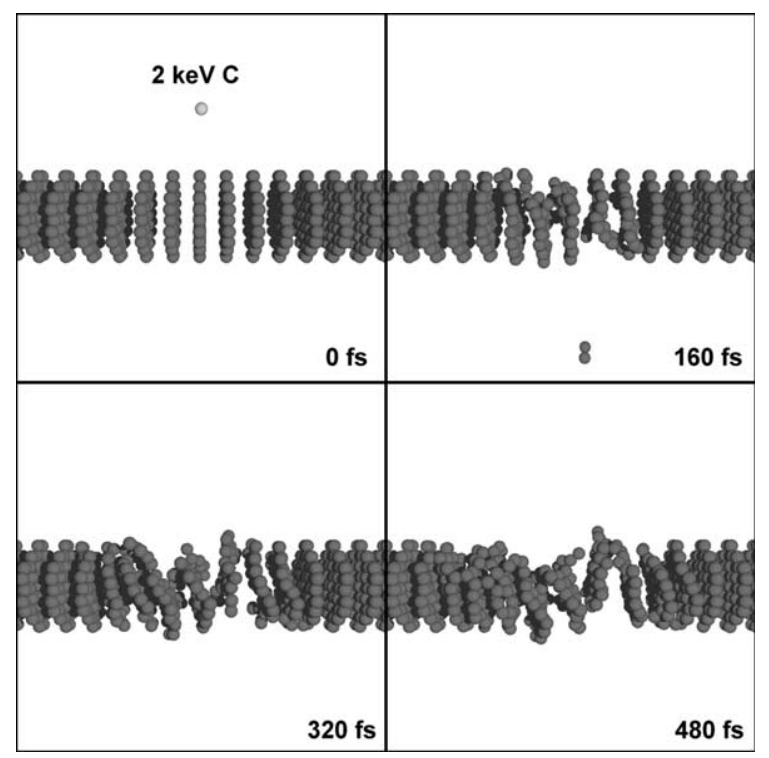

Fig. 2. Time sequence of the interaction of a $2 \mathrm{keV}$ carbon ion with a graphite cylinder. 
quite well and even though the damage here seems to be quite catastrophic the general shape of the structure remains intact. It is quite conceivable that a real DNA molecule could either recover from this impact also. One of the reasons that heavy ions have been used in radiation therapy is that they create more substantial damage than a proton and have a similar energy loss profile. Again the principal damaging mechanism is via the bond breaking mechanism and there is a greater density of energy loss to the electronic system in the case of carbon irradiation than with protons [9]. Consequently the same arguments as for protons might be considered to apply for $\mathrm{C}$ irradiation as well and the greater part of the damage process results in simple defects, some of which will be relatively easy to repair.

Carbon atoms are used in heavy ion irradiation for their bio-compatibility. To increase the nuclear interaction of the impacting particles so that create more substantial damage to the carbon cylinders would require the interaction of higher mass particles which would not necessarily have the same compatibility. The alternative is to make use of cluster or molecular species. The purpose, then, of this thought experiment is to investigate the potential change in nuclear displacement damage caused by the interaction of a large energetic carbon molecule. Fullerene is a well defined robust carbon molecule containing 60 carbon atoms. As the molecule consists of carbon atoms its slowing down and energy loss process at high energy should be similar to that of a similar velocity carbon particle and the principle slowing down mechanism should be via collision with electrons and as carbon is very light in comparison to an electron the molecule should remain mostly "intact" although the binding electrons may well be lost during the slowing down process. Even in this situation we should still expect the molecule to travel together in an unbound state and hence up to 60 atoms should still collide with the carbon cylinder simultaneously once the energy drops enough for collisions with nuclei to become probable.

Fig. 3 shows what happens if a $120 \mathrm{keV} \mathrm{C}_{60}$ (same velocity as a $2 \mathrm{keV} \mathrm{C}$ ) impacts with the carbon cylinder structure. Substantial damage occurs and the structure is completely destroyed. It

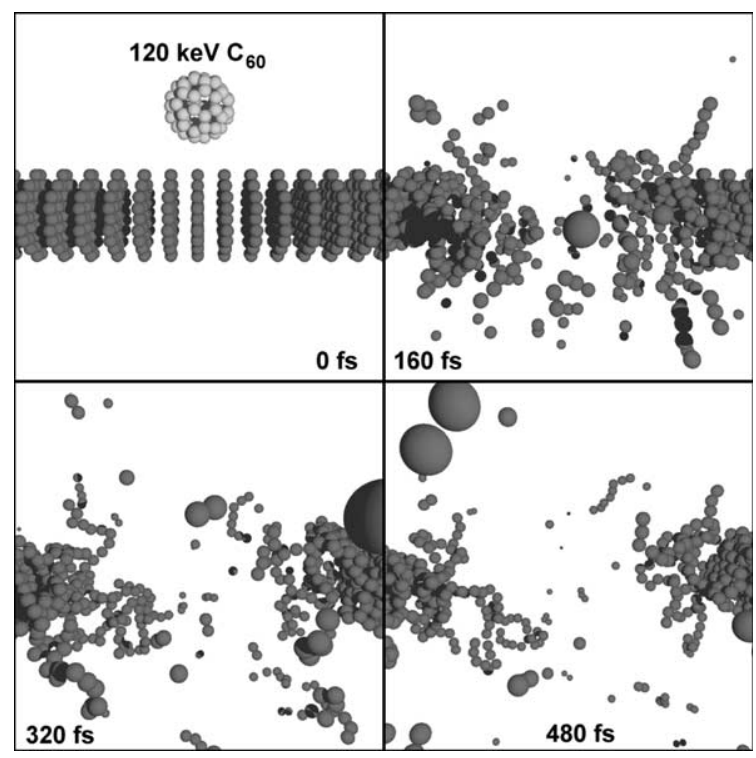

Fig. 3. Time sequence of the interaction of a $120 \mathrm{keV} \mathrm{C}_{60}$ fullerene with a graphite cylinder.

is extremely unlikely that this damage will be in anyway repairable. At first sight, then, our thought experiment indicates that molecule or cluster irradiation could produce more substantial damage than that of either proton or carbon irradiation. However, for this to become a viable therapy there are many problems least of all the generation of a beam of sufficiently high velocity. To get the same depth of penetration as $\mathrm{C}$ at $300 \mathrm{MeV}$ we can expect to need to produce a $\mathrm{C}_{60}$ beam of $18 \mathrm{GeV}$. This does not seem to be very likely for a large number of patients, but with $60 \mathrm{C}$ particles it should be possible to obtain a molecular beam with multiple charges thus lowering the terminal voltage needed. However, there is also the problem of how well does the molecule propagate through the carbon cylinders at high energy? To be used in a similar way as proton and carbon therapy we must be able to deliver the $\mathrm{C}_{60}$ still largely intact deep into the irradiated material. In particular we should be able to pass a $\mathrm{C}_{60}$ molecule through our carbon cylinder structure at high energies without it breaking up or causing substantial damage. Hence the next part of our thought experiment is to investigate the behaviour of the fullerene when it encounters the cylinder at high energy. 


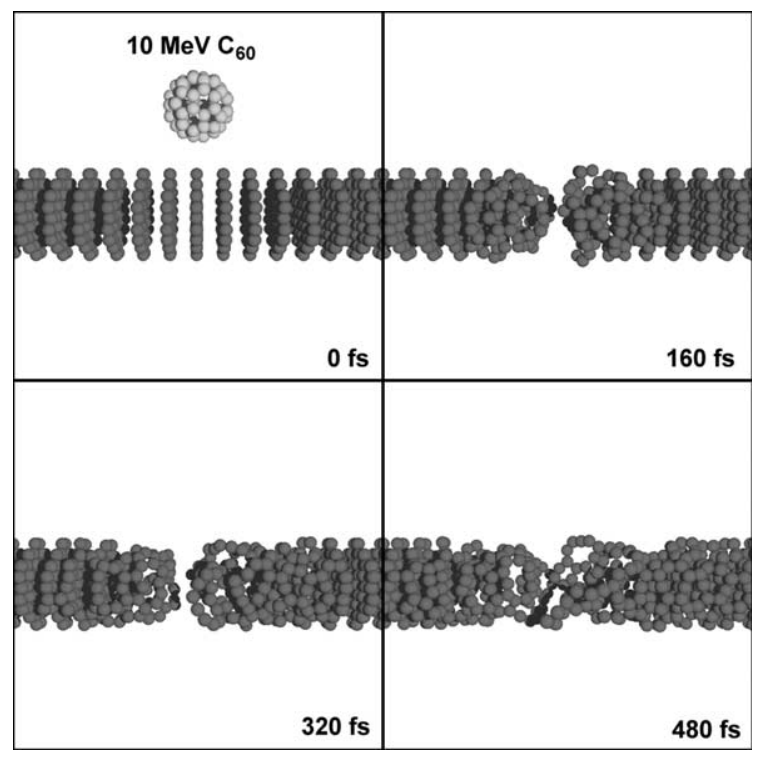

Fig. 4. Time sequence of the interaction of a $10 \mathrm{MeV} \mathrm{C}_{60}$ fullerene with a graphite cylinder.

In Fig. 4 the impact of a $10 \mathrm{MeV} \mathrm{C}_{60}$ with the cylinder structure is shown. It still seems to be creating a relatively large amount of damage to the structure similar to that generated by the $2 \mathrm{keV} \mathrm{C}$ impact. In contrast a $200 \mathrm{keV} \mathrm{C}$ - similar velocity as the $10 \mathrm{MeV} \mathrm{C}_{60}$ - passes without any substantial interaction in the vast majority of encounters with the cylinder.

By tracking the "camera" at a constant velocity with the $\mathrm{C}_{60}$ we can see what happens to it as it passes through the cylinder structure, this is shown in Fig. 5. As we can see it does not remain intact. It fragments into a number of smaller clusters. This is quite typical of all of the impacts (35) we tried at this energy. This indicates that it will probably be quite difficult to deliver the $\mathrm{C}_{60}$ to a buried cylinder at an energy of the order $200 \mathrm{keV}$. This suggests that there probably will be no advantage in using a large molecule for deep penetration as the molecule will almost certainly fragment well before it slows down enough to deposit substantial energy in nuclear collisions.

However, the fragmented sub-clusters will themselves be able to deposit substantial energy simultaneously to the cylinder. If we make the cluster smaller can we still enhance the damage by

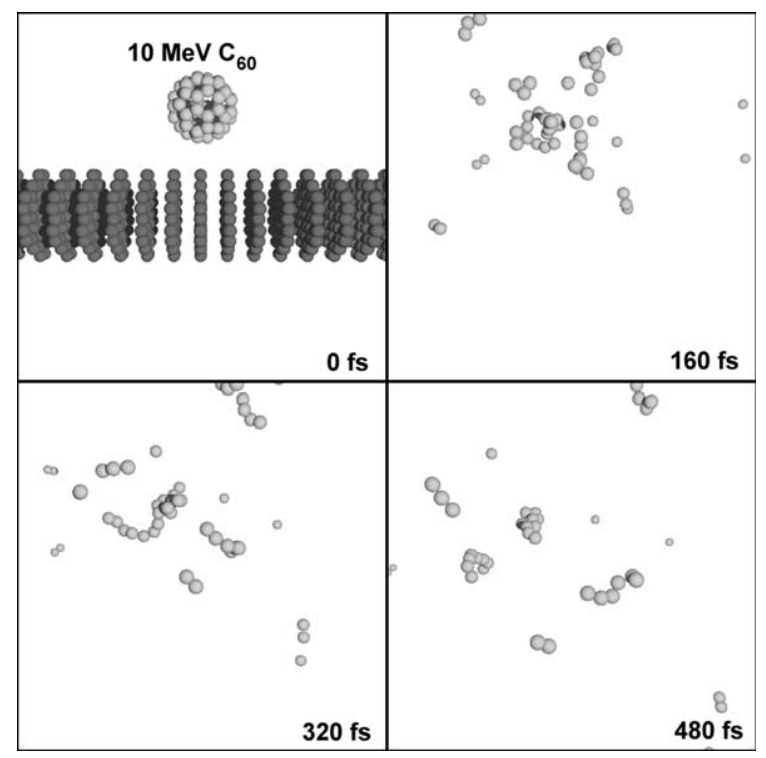

Fig. 5. Time sequence of the interaction of a $10 \mathrm{MeV} \mathrm{C}_{60}$ fullerene with a graphite cylinder. The camera is moving with same velocity as the initial velocity of the fullerene.

striking the same area simultaneously and still be able to pass through the cylinder at high energy?

To help answer this we show, in Fig. 6, a simulation of a $\mathrm{C}_{6}$ (in a benzene like arrangement)

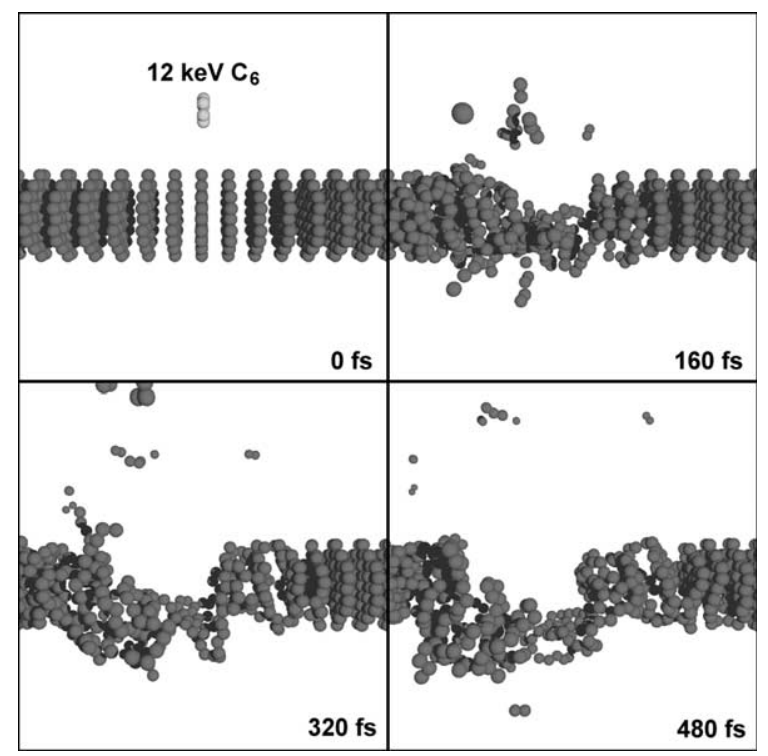

Fig. 6. Time sequence of the interaction of a $12 \mathrm{keV} \mathrm{C}_{6}$ ring molecule with a graphite cylinder. 
hitting edge on (we did try a very limited number of different orientations and this result is typical of those we found) with an energy of $12 \mathrm{keV}$ (same velocity the $2 \mathrm{keV} \mathrm{C}$ ). It can be seen that it still causes substantial break up of the structure - not as dramatic as the impact of $\mathrm{C}_{60}$, but the structure appears to be damaged enough that it is hard to believe that it would be able to recover.

Fig. 7 shows that we can pass the molecule relatively harmlessly through the cylinder without doing any significant damage to the structure at high energy. The example here was tried at $1 \mathrm{MeV}$, the same velocity as the $10 \mathrm{MeV} \mathrm{C}_{60}$. However if we look to see the influence of the interaction on the $\mathrm{C}_{6}$ molecule we find that it is no longer intact see Fig. 8 - and it has been broken up so that only a $\mathrm{C}_{4}$ cluster remains intact. It is quite likely that the internal energy of this fragment is such that it will not remain intact for long and may well fragment further.

In conclusion then, we have shown that molecules and cluster impacts on our carbon cylinder will certainly create much more substantial damage then a single atomic particle. The implication is that this will also be true for irradiation of DNA

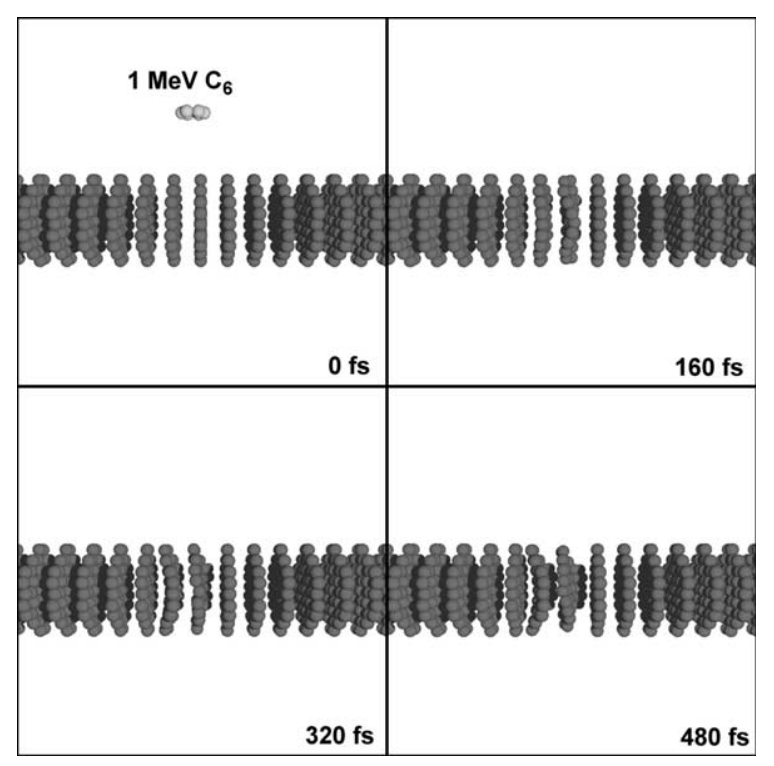

Fig. 7. Time sequence of the interaction of a $1 \mathrm{MeV} \mathrm{C}_{6}$ ring molecule with a graphite cylinder.

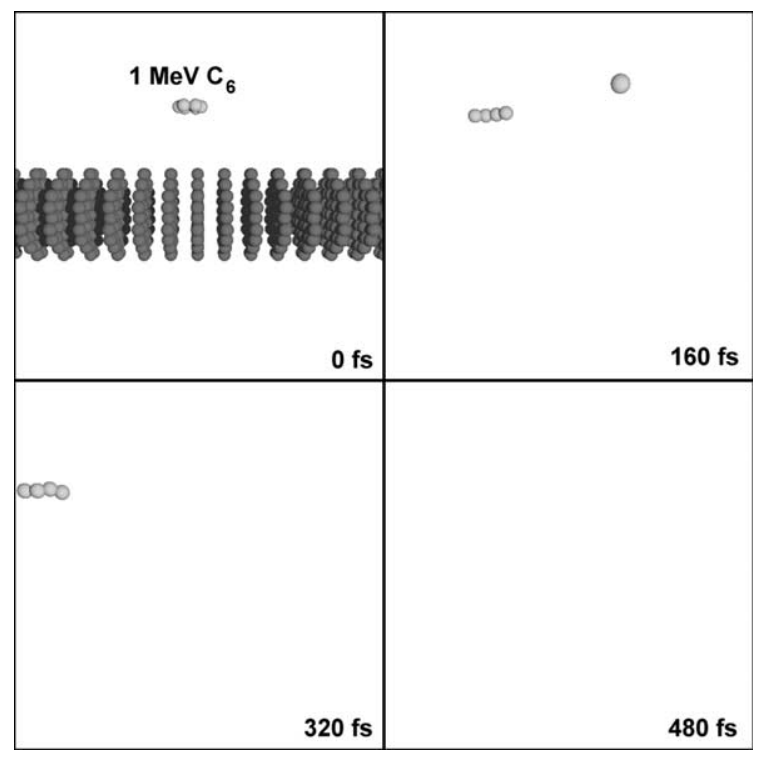

Fig. 8. Time sequence of the interaction of a $1 \mathrm{MeV} \mathrm{C}_{6}$ ring molecule with a graphite cylinder. The camera is moving at the same velocity as the initial velocity of the molecule.

in a cell. The problem is that delivery of intact molecule deep inside the material will probably not be possible. It would seem quite likely that the clusters or molecules will fragment at high energies long before they are in a position to transfer substantial energy to the DNA strands. In this case it would appear that there would be no advantage in the use of molecular species for therapy.

\section{References}

[1] See for example web pages of the National Institute of Radiological Sciences in Japan at http://www.nirs.go.jp and those of at the Midwest Proton Radiation Institute at Indiana University at http://www.iucf.indiana.edu/MPRI.

[2] D.W. Miller, Med. Phys. 22 (11) (1995) 1943.

[3] C.F. Serago, Med. Phys. 22 (12) (1995) 2111.

[4] M. Durante, S. Yamada, K. Ando, Y. Furusawa, Int. J. Radiat. Biol. 47 (2000) 793.

[5] B.J. Sealy, R.P. Webb, M. Breese (Eds.), Proceedings of 7th European Conference on Accelerators in Applied Research and Technology, Nucl. Instrum. and Meth. B $188(1-4)(2002) 1$.

[6] The discussion at the workshop was led by N. Kirkby of the University of Surrey after an invited talk by $M$. Folkard of the Gray Laboratory. 
[7] H. Nikjoo, S. Uehara, I.G. Khvostunov, F.A. Cucinotta, W.E. Wilson, D.T. Goodhead, Phys. Med. 17 (1) (2000) 38.

[8] A. Ottolenghi, M. Merzagora, L. Tallone, M. Durante, H.G. Paretzke, W.E. Wilson, Radiat. Environ. Biophys. 34 (1995) 239.

[9] M. Kraemer, M. Scholz, Phys. Med. Biol. 45 (2000) 3319.
[10] R. Smith, D.E. Harrison Jr., B.J. Garrison, Phys. Rev. B40 (1989) 93.

[11] D.W. Brenner, Phys. Rev. B 42 (15) (1990) 9458.

[12] R. Smith, K. Beardmore, Thin Solid Films 272 (1996) 255.

[13] See for example http://www.accessexcellence.org/AB/GG/ structure.html and associated links. 